\title{
Kaon optical potential in nuclei and kaon condensation in neutron star
}

\section{Y. Ryu*}

Department of Physics, Sungkyunkwan University, Suwon 440-746, Korea

E-mail: 'cyryuécolor. skku acc.

\section{H. Hyun}

Institute of Basic Science, Sungkyunkwan University, Suwon 440-746, Korea

School of Physics, Seoul National University, Seoul 151-742, Korea

E-mail:

\section{S. W. Hong}

Department of Physics, Sungkyunkwan University, Suwon 440-746, Korea

E-mail: 'swhong@ skku.ac. kris

\section{B. T. Kim}

Department of Physics, Sungkyunkwan University, Suwon 440-746, Korea

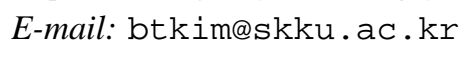

Recent experiments at KEK, BNL, and DAФNE revealed very interesting peaks that might be interpreted as deeply bound kaonic nuclear systems, which were predicted by Akaishi and Yamazaki. If those peaks are indeed found to be deeply bound kaonic nuclear states, it implies that the real part of the kaon optical potential in nuclei can be very large. Here we consider this possibility of very large kaon optical potential in the framework of a modified quark-meson coupling model. We then apply the modified quark-meson coupling model to the neutron star matter. It is found that the composition of matter is very sensitive to the interaction strength of kaons with matter. The presense of kaon condensation changes drastically the population of octet baryons and leptons. We compare the results from the modified quark-meson coupling model with those of quantum hadrodynamics. The kaon condensation starts to take place at lower densities with the modified quark-meson coupling model.

29th Johns Hopkins Workshop on current problems in particle theory: strong matter in the heavens 1-3 August

Budapest

\footnotetext{
* Speaker.
} 


\section{Introduction}

The interior of a neutron star is believed to have great diversity. At densities around the saturation density $\rho_{0}$, the matter is composed of nucleons, electrons and muons, and it is mostly populated by the neutrons to satisfy the $\beta$-equilibrium, charge neutrality and Pauli blocking. At densities higher than $\rho_{0}$, maybe at $\rho \geq 2 \rho_{0}$, the situation becomes quite unclear. Many possibilities have been proposed; creation of hyperons [i-1] quark deconfinement $\left[\bar{s}_{1}, \overline{6}_{n}, 1\right]$ of neutron star properties such as mass, radius, and temperature, understanding them still confronts many problems and uncertainties due to lack of our knowledge about the state of matter at high densities.

In this work, we consider the possibility of hyperon creation and kaon condensation in the neutron star matter. The masses and energies of the hyperons and kaons in medium are sensitive to their interactions with surrounding matter. In the meson-exchange picture, meson-hyperon and meson-kaon coupling constants determine the strength of these interactions. The meson-hyperon coupling constants may be determined from the binding energies of hyperons in hypernuclei, for instance. The meson-kaon coupling constants have been studied by using the kaon-nucleon scat-

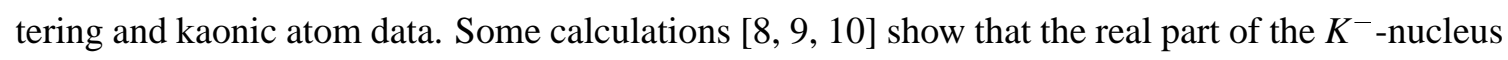
optical potential $U_{K^{-}}$is shallow $\left(U_{K^{-}} \approx-50 \mathrm{MeV}\right)$, but some other calculations suggest that $U_{K^{-}}$

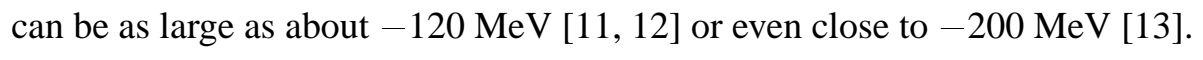

Recently, Akaishi and Yamazaki predicted the existence of deeply bound kaonic nuclei [i] 1 ind, in which $U_{K^{-}}$at normal density $\rho_{0}$ is estimated to be about $-120 \mathrm{MeV}$. Observations of the claimed

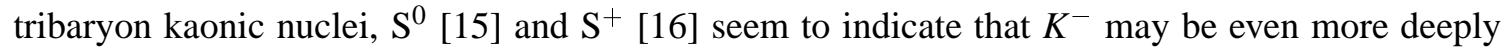
bound in a nucleus than the theoretical prediction [1]

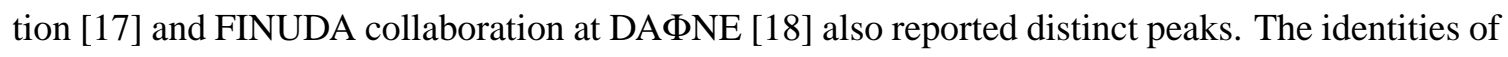
these peaks need to be studied further experimentally and theoretically. However, in this work, we consider the possibility of deep optical potential of kaons and apply such deep potentials to neutron star matter to explore the consequences in the equation of state $(\mathrm{EoS})$ of dense matter and neutron star properties.

We employ the modified quark-meson coupling (MQMC) model [1] dense matter. Nucleons and hyperons in the baryon octet are described as MIT bags. The bag constant $B_{B}$ and phenomenological constant $Z_{B}$ for baryon $B$ are determined to reproduce the free mass of each baryon. Coupling constants between $(u, d)$-quarks in the bags and $(\sigma, \omega, \rho)$-mesons are adjusted to produce the saturation density $\rho_{0}=0.17 \mathrm{fm}^{-3}$, binding energy per a nucleon $E_{b} / A=$ $16 \mathrm{MeV}$ and symmetry energy at the saturation $a_{\text {sym }}=32.5 \mathrm{MeV}$. Since the interaction between the $s$-quark and mesons are not well known, we adopt the standard quark counting rule and assume the $s$-quark is decoupled to $(\sigma, \omega, \rho)$-mesons. To take into account the interactions between $s$ quarks, we introduce $\sigma^{*}\left(f_{0}(980)\right)$ and $\phi(1020)$ mesons following Ref. [20 $\left.20-1\right]$. We assume the kaon as a point particle. This treatment allows us to use $U_{K^{-}}$as an input to fix the kaon-meson coupling constants. In our model the real part of the optical potential can be written as $U_{K^{-}}=-\left(g_{\sigma K} \sigma\left(\rho_{0}\right)+\right.$ $\left.g_{\omega K} \omega\left(\rho_{0}\right)\right)$, where $\sigma\left(\rho_{0}\right)$ and $\omega\left(\rho_{0}\right)$ are the values of the meson fields at $\rho_{0}$. By using the value of $g_{\omega K}$ given by the quark counting rule, we can determine $g_{\sigma K}$ for each given value of $U_{K^{-}}$. Once the parameters of the model are fixed, the composition profile of neutron star matter can be obtained 
from $\beta$-equilibrium and charge neutrality. We find that the composition of neutron star matter changes dramatically depending on the value of $U_{K^{-}}$. To investigate the model dependence we employ quantum hadrodynaics (QHD) model and compare the results from QHD with those from the MQMC model. We find the onset density of the kaon condensation and the composition of matter at high densities are substantially different.

\section{Theory}

In this section we briefly sketch the MQMC and QHD models by giving the model Lagrangians. Then coupling constants in each model are determined to produce the same saturation properties; the saturation density, the binding energy, the symmetry energy, the nucleon effective mass and the compression modulus. Finally we describe how the variables that determine the EoS of the neutron star matter can be solved self-consistently.

\subsection{Models}

The model Lagrangian comprises the octet baryon, exchange meson, lepton and kaon terms as $\mathscr{L}_{\text {tot }}=\mathscr{L}_{B}+\mathscr{L}_{M}+\mathscr{L}_{l}+\mathscr{L}_{K}$. Octet baryon, exchange meson and lepton terms in the mean field approximation can be written as

$$
\begin{aligned}
\mathscr{L}_{B} & =\sum_{B} \bar{\psi}_{B}\left[i \gamma \cdot \partial-m_{B}^{*}\left(\sigma, \sigma^{*}\right)-\gamma^{0}\left(g_{\omega B} \omega_{0}+g_{\phi B} \phi_{0}+\frac{1}{2} g_{\rho B} \tau_{z} \rho_{03}\right)\right] \psi_{B} \\
\mathscr{L}_{M} & =-\frac{1}{2} m_{\sigma}^{2} \sigma^{2}-\frac{1}{2} m_{\sigma^{*}}^{2} \sigma^{* 2}+\frac{1}{2} m_{\omega}^{2} \omega_{0}^{2}+\frac{1}{2} m_{\phi}^{2} \phi_{0}^{2}+\frac{1}{2} m_{\rho}^{2} \rho_{03}^{2}, \\
\mathscr{L}_{l} & =\sum_{l} \bar{\psi}_{l}\left(i \gamma \cdot \partial-m_{l}\right) \psi_{l},
\end{aligned}
$$

where $B$ denotes the sum over all the baryon octet $\left(p, n, \Lambda, \Sigma^{+}, \Sigma^{0}, \Sigma^{-}, \Xi^{0}, \Xi^{-}\right)$, and $l$ stands for the sum over the free electrons and muons $\left(e^{-}, \mu^{-}\right), \sigma, \omega$ and $\rho$ mesons mediate the interactions between the non-strange light quarks $(u$ and $d) . \sigma^{*}$ and $\phi$ mesons are introduced to take into account the interactions between $s$ quarks. $\mathscr{L}_{B}$ is of the identical form for both the MQMC and the QHD models, but the two models differ in the expression for the effective baryon mass $m_{B}^{*}$.

\section{MQMC}

In the MQMC model, a baryon is a composite system with quarks in a spherical bag, and thus its mass is given in terms of bag parameters and quark energy. The effective mass of a baryon in

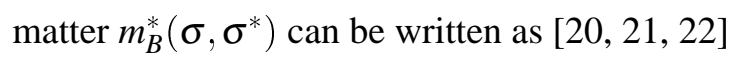

$$
m_{B}^{*}=\sqrt{E_{B}^{2}-\sum_{q}\left(\frac{x_{q}}{R}\right)^{2}} .
$$

The bag energy of a baryon is given by

$$
E_{B}=\sum_{q} \frac{\Omega_{q}}{R}-\frac{Z_{B}}{R}+\frac{4}{3} \pi R^{3} B_{B},
$$

where $B_{B}$ and $Z_{B}$ are the bag constant and a phenomenological constant for the zero-point motion of a baryon $B$, respectively. $\Omega_{q}=\sqrt{x_{q}^{2}+\left(R m_{q}^{*}\right)^{2}}$, where $m_{q}^{*}\left(=m_{q}-g_{\sigma}^{q} \sigma-g_{\sigma^{*}}^{q} \sigma^{*}\right)$ is the effective 
mass of a quark whose free mass is $m_{q}$. We take $m_{q}=0$ for $q=u, d$ and $m_{q}=150 \mathrm{MeV}$ for $q=s$. $x_{q}$ is determined from the boundary condition on the bag surface $r=R$,

$$
j_{0}\left(x_{q}\right)=\beta_{q} j_{1}\left(x_{q}\right),
$$

where $\beta_{q}=\sqrt{\frac{\Omega_{q}-R m_{q}^{*}}{\Omega_{q}+R m_{q}^{*}}}$. In the MQMC model, the bag constant $B_{B}$ is assumed to depend on density. In this work, we extend the form used in [i1 $\left[\begin{array}{l}1 \\ 9\end{array}\right]$ to include the contribution from $\sigma^{*}$ as

$$
B_{B}\left(\sigma, \sigma^{*}\right)=B_{B 0} \exp \left\{-4 g^{\prime B}\left(\sum_{q=u, d} n_{q} \sigma+\left(3-\sum_{q=u, d} n_{q}\right) \sqrt{2} \sigma^{*}\right) / m_{B}\right\},
$$

where $m_{B}$ is the free mass of the baryon $B$. Note that $\sigma$ meson couples to $u$ and $d$ quarks only, and $\sigma^{*}$ meson to $s$ quark only. The factor $\sqrt{2}$ in front of $\sigma^{*}$ is introduced due to $\operatorname{SU}(6)$ symmetry.

\section{QHD}

In the QHD model, a baryon is treated as a point particle, and thus its effective mass is simply written as

$$
m_{B}^{*}=m_{B}-g_{\sigma B} \sigma-g_{\sigma^{*} B} \sigma^{*} .
$$

In order to reproduce the same saturation properties as the MQMC, self-interactions of the $\sigma$-field

$$
U_{\sigma}^{\mathrm{QHD}}=\frac{1}{3} g_{2} \sigma^{3}+\frac{1}{4} g_{3} \sigma^{4}
$$

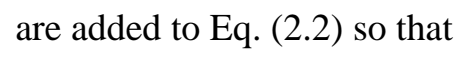

$$
\mathscr{L}_{M}^{\mathrm{QHD}}=\mathscr{L}_{M}-U_{\sigma}^{\mathrm{QHD}} .
$$

The baryon and the lepton Lagrangians for the QHD model take the form given by Eqs. (ㄱ.2.1.1) and $(\overline{2} \cdot \overline{3})$.

\section{Kaon}

The effective Lagrangian for the kaon can be expressed as [느르.

$$
\mathscr{L}_{K}=D_{\mu}^{*} K^{*} D^{\mu} K-m_{K}^{* 2} K^{*} K
$$

where $D_{\mu}=\partial_{\mu}+i g_{\omega K} \omega_{\mu}-i g_{\phi K} \phi_{\mu}+i \frac{1}{2} g_{\rho K} \vec{\tau} \cdot \vec{\rho}_{\mu}$. In this work we treat the kaon as a point particle in both MQMC and QHD models, and its effective mass is given by

$$
m_{K}^{*}=m_{K}-g_{\sigma K} \sigma-g_{\sigma^{*} K} \sigma^{*} .
$$

The equation of motion for a kaon is obtained as

$$
\left[D_{\mu} D^{\mu}+m_{K}^{* 2}\right] K(x)=0 .
$$

In uniform infinite matter, the kaon field $K(x)$ can be written as a plane wave. Substituting the plane wave solution into the equation of motion, we obtain the dispersion relation for the anti-kaon

$$
\omega_{K}=m_{K}^{*}-g_{\omega K} \omega_{0}+g_{\phi K} \phi_{0}-g_{\rho K} I_{3 K} \rho_{03},
$$

where $I_{3 K}$ is the isospin third component of the anti-kaon. (In this work it is the anti-kaon $K^{-}$that plays an important role, but we refer to anti-kaons just as kaons for brevity.) 


\begin{tabular}{|c|c|c|c|}
\hline$B$ & $m_{B}(\mathrm{MeV})$ & $B_{B 0}^{1 / 4}(\mathrm{MeV})$ & $Z_{B}$ \\
\hline$N$ & 939.0 & 188.1 & 2.030 \\
\hline$\Lambda$ & 1115.6 & 197.6 & 1.926 \\
\hline$\Sigma^{+}$ & 1189.4 & 202.7 & 1.829 \\
\hline$\Sigma^{0}$ & 1192.0 & 202.9 & 1.826 \\
\hline$\Sigma^{-}$ & 1197.3 & 203.3 & 1.819 \\
\hline$\Xi^{0}$ & 1314.7 & 207.6 & 1.775 \\
\hline$\Xi^{-}$ & 1321.3 & 208.0 & 1.765 \\
\hline
\end{tabular}

Table 1: Bag constants $B_{B 0}$ and phenomenological constants $Z_{B}$ for octet baryons obtained to reproduce the free mass of each baryon. Bag radius $R_{0}=0.6 \mathrm{fm}$ for all baryon octet and the bare mass of quarks, $m_{u(d)}=0$ $\mathrm{MeV}$ and $m_{s}=150 \mathrm{MeV}$.

\begin{tabular}{|c|c|c|c|c|c|}
\hline$g_{\sigma}^{q}$ & $g_{\omega}^{q}$ & $g_{\sigma}^{\prime B}$ & $g_{\rho}^{q}$ & $m_{N}^{*} / m_{N}$ & $K(\mathrm{MeV})$ \\
\hline 1.0 & 2.71 & 2.27 & 7.88 & 0.78 & 285.5 \\
\hline
\end{tabular}

Table 2: The coupling constants for $(u, d)$-quarks and $(\sigma, \omega, \rho)$-mesons in the MQMC model to reproduce the binding energy $E_{b} / A=16 \mathrm{MeV}$ at the saturation density $0.17 \mathrm{fm}^{-3}$ and symmetry energy $a_{\text {sym }}=32.5$ $\mathrm{MeV} . m_{N}^{*} / m_{N}$ and $K$ are the ratio of the effective mass to the free mass of the nucleon and the compression modulus at the saturation density, respectively.

\subsection{Model parameters}

\section{MQMC}

In the MQMC model, MIT bag parameters $B_{B 0}$ and $Z_{B}$ are determined to reproduce the free mass of a baryon $B,\left.m_{B}^{*}\right|_{\rho=0}=m_{B}$ with the minimization condition $\left.\frac{\partial m_{B}}{\partial R}\right|_{R=R_{0}}=0$ at a given free bag radius $R_{0}$, which we choose as $R_{0}=0.6 \mathrm{fm}$. Table $i_{-1}^{1}$ shows the bag parameters $B_{B 0}$ and $Z_{B}$ for the octet baryons.

Three saturation conditions $\rho_{0}, E_{b} / A$, and $a_{\text {sym }}$ could determine three quark-meson coupling constants $g_{\sigma}^{u, d}, g_{\omega}^{u, d}$ and $g_{\rho}^{u, d}$, assuming $u$ and $d$ quarks are identical particles in an isodoublet. The MQMC model, however, introduces an additional constant $g_{\sigma}^{\prime B}$, and thus four coupling constants cannot be determined uniquely. We fix $g_{\sigma}^{q}=1$, and adjust the remaining three constants to meet the three conditions. Obtained coupling constants are given in Table $\bar{q}$ together with the effective mass ratio of the nucleon $m_{N}^{*} / m_{N}$ and the compression modulus $K$. Table 2 shows $m_{N}^{*}$ and $K$ are reproduced within reasonable ranges; $m_{N}^{*}=(0.7 \sim 0.8) m_{N}$ and $K=(200 \sim 300) \mathrm{MeV}$. The coupling constants between $s$-quarks and mesons cannot be determined from the conditions at the saturation density. In principle, experimental data from hypernuclei, kaon-nucleus scattering and kaonic atom could be used to determine meson-hyperon coupling constants. However, these coupling constants are not well known yet, and for simplicity we assume that the quark counting rule hold and that the $s$ quark does not interact with $u$ and $d$ quarks. Then we have

$$
g_{\sigma}^{s}=g_{\omega}^{s}=g_{\rho}^{s}=0 .
$$

Applying the quark counting rule to the meson-baryon coupling constants which appear in the 


\begin{tabular}{|l|c|c|c|c|}
\hline$g_{\sigma N}$ & $g_{\omega N}$ & $g_{\rho N}$ & $g_{2}\left(\mathrm{fm}^{-1}\right)$ & $g_{3}$ \\
\hline 8.06 & 8.19 & 7.88 & 12.139 & 48.414 \\
\hline
\end{tabular}

Table 3: The meson-nucleon coupling constants and the coefficients of the $\sigma$-meson self interaction terms in the QHD model. They reproduce the five saturation properties $\rho_{0}, E_{b}, a_{\text {sym }}, m_{N}^{*}$ and $K$ as given in Table 2 .

\begin{tabular}{|c|c|c|c|c|c|}
\hline$\left|U_{K^{-}}\right|(\mathrm{MeV})$ & 80 & 100 & 120 & 140 & 160 \\
\hline$g_{\sigma K}(\mathrm{MQMC})$ & 1.25 & 2.01 & 2.75 & 3.50 & 4.25 \\
\hline$g_{\sigma K}(\mathrm{QHD})$ & 1.26 & 2.04 & 2.82 & 3.61 & 4.39 \\
\hline
\end{tabular}

Table 4: $g_{\sigma K}$ determined for several $U_{K^{-}}$values in the MQMC and the QHD models.

model Lagrangian, we obtain the relations

$$
\begin{aligned}
g_{\omega}^{q} & =\frac{1}{3} g_{\omega N}=\frac{1}{2} g_{\omega \Lambda}=\frac{1}{2} g_{\omega \Sigma}=g_{\omega \Xi} \\
g_{\rho}^{q} & =g_{\rho N}=g_{\rho \Sigma}=g_{\rho \Xi}, \quad g_{\rho \Lambda}=0, \\
g_{\phi}^{s} & =g_{\phi \Lambda}=g_{\phi \Sigma}=\frac{1}{2} g_{\phi \Xi}
\end{aligned}
$$

where $g_{\phi}^{s}=\sqrt{2} g_{\omega}^{u, d}$ from the SU(6) symmetry. The quark-meson coupling constants given in Ta-

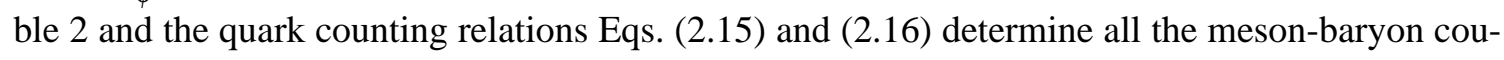
pling of the MQMC model.

\section{QHD}

In the QHD model, $g_{\sigma N}$ and $g_{\omega N}$ are adjusted to yield $\rho_{0}$ and $E_{b}$, and $g_{\rho N}$ is fitted to produce $a_{\text {sym }} \cdot g_{2}$ and $g_{3}$ in $U_{\sigma}^{\mathrm{QHD}}$ are fixed to reproduce $m_{N}^{*}$ and $K$ values of the MQMC model in Table $\stackrel{\overline{2}}{\stackrel{2}{*}}$. Thus determined coupling constants are given in Table $\underset{\text { s. }}{\overline{1}}$. As in the MQMC model, hyperon-meson coupling constants in the QHD are fixed by the quark counting rule and the SU(6) symmetry.

\section{Kaon}

There are 5 meson-kaon coupling constants, $g_{\sigma K}, g_{\omega K}, g_{\rho K}, g_{\sigma^{*} K}$ and $g_{\phi K} . g_{\omega K}$ and $g_{\rho K}$ are fixed from the quark counting rule; $g_{\omega K}=g_{\omega}^{q}$ and $g_{\rho K}=g_{\rho}^{q} . g_{\sigma^{*} K}$ can be fixed from $f_{0}(980)$ decay

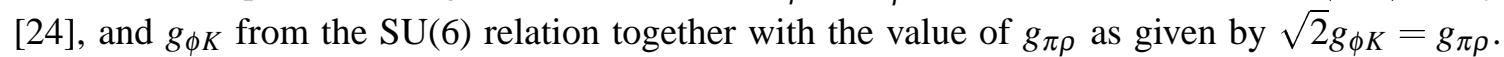
$g_{\sigma^{*} K}$ and $g_{\phi K}$ thus fixed are 2.65 and 4.27, respectively. The remaining coupling constant, $g_{\sigma K}$, can be related to the real part of the optical potential of a kaon at the saturation density; $U_{K^{-}}=$ $-\left(g_{\sigma K} \sigma+g_{\omega K} \omega_{0}\right) \cdot g_{\sigma K}$ values thus determined are summarized for several $U_{K^{-}}$values in Table $\bar{i}_{\text {-. }}$

\subsection{EoS of neutron star matter}

To obtain the EoS, we need to determine the variables at each density: 5 meson fields $\left(\sigma, \omega, \rho, \sigma^{*}, \phi\right.$ ), 8 octet baryon densities, 2 lepton densities and the kaon density $\rho_{K} .5$ meson fields can be determined from their equations of motion:

$$
m_{\sigma}^{2} \sigma+\frac{\partial}{\partial \sigma} U_{\sigma}^{\mathrm{QHD}}=\sum_{B} g_{\sigma B} C_{B}(\sigma) \frac{2 J_{B}+1}{2 \pi^{2}} \int_{0}^{k_{B}} \frac{m_{B}^{*}}{\left[k^{2}+m_{B}^{* 2}\right]^{1 / 2}} k^{2} d k+g_{\sigma K} \rho_{K},
$$




$$
\begin{gathered}
m_{\sigma^{*}}^{2} \sigma^{*}=\sum_{B} g_{\sigma^{*} B} C_{B}\left(\sigma^{*}\right) \frac{2 J_{B}+1}{2 \pi^{2}} \int_{0}^{k_{B}} \frac{m_{B}^{*}}{\left[k^{2}+m_{B}^{* 2}\right]^{1 / 2}} k^{2} d k+g_{\sigma^{*} K} \rho_{K}, \\
m_{\omega}^{2} \omega_{0}=\sum_{B} g_{\omega B}\left(2 J_{B}+1\right) k_{B}^{3} /\left(6 \pi^{2}\right)-g_{\omega K} \rho_{K} \\
m_{\phi}^{2} \phi_{0}=\sum_{B} g_{\phi B}\left(2 J_{B}+1\right) k_{B}^{3} /\left(6 \pi^{2}\right)+g_{\phi K} \rho_{K} \\
m_{\rho}^{2} \rho_{03}=\sum_{B} g_{\rho B} I_{3 B}\left(2 J_{B}+1\right) k_{B}^{3} /\left(6 \pi^{2}\right)-g_{\rho K} I_{3 K} \rho_{K},
\end{gathered}
$$

where $J_{B}$ and $I_{3 B}$ are the spin and the isospin projection, respectively, and $k_{B}$ is the Fermi momentum of the baryon species $B$. In Eq. (2.17), $U_{\sigma}^{\mathrm{QHD}}$ is only for the QHD model, and is not to be included for the MQMC model. $C_{B}(\sigma)$ and $C_{B}\left(\sigma^{*}\right)$ are determined from the relations $g_{\sigma B} C_{B}(\sigma)=-\frac{\partial m_{B}^{*}}{\partial \sigma}$ and $g_{\sigma^{*} B} C_{B}\left(\sigma^{*}\right)=-\frac{\partial m_{B}^{*}}{\partial \sigma^{*}}$. $\beta$-equilibrium of the baryons gives us the following 7 relations

$$
\begin{gathered}
\mu_{n}=\mu_{\Lambda}=\mu_{\Sigma^{0}}=\mu_{\Xi^{0}}, \\
\mu_{n}+\mu_{e}=\mu_{\Sigma^{-}}=\mu_{\Xi^{-}}, \\
\mu_{n}-\mu_{e}=\mu_{p}=\mu_{\Sigma^{+}},
\end{gathered}
$$

where the chemical potential of a baryon $B$ is given by $\mu_{B}=\sqrt{k_{B}^{2}+m_{B}^{* 2}\left(\sigma, \sigma^{*}\right)}+g_{\omega B} \omega_{0}+g_{\phi B} \phi_{0}+$ $g_{\rho B} I_{3 B} \rho_{03}$. For non-interacting leptons, the chemical potential of a lepton $l$ is simply written as $\mu_{l}=\sqrt{k_{l}^{2}+m_{l}^{2}}$. The lepton equilibrium condition

$$
\mu_{e}=\mu_{\mu}
$$

determines the density of muons. At a density where the condition

$$
\omega_{K}=\mu_{n}-\mu_{p}
$$

is satisfied, kaons are condensed and the kaon density $\rho_{K}$ becomes non-zero. Finally, charge neutrality is expressed as

$$
\sum_{B} q_{B} \rho_{B}-\rho_{K}-\rho_{e}-\rho_{\mu}=0,
$$

where $q_{B}$ is the charge of baryon species $B$ and $\rho_{B}$ is the number density of $B$. Solving the Eqs. (2.172 $\left.25^{\prime}\right)$ self-consistently and simultaneously together with the quark eigenvalue equation Eq. $(\overline{2}-6)$, one can determine the 16 variables uniquely. Once these 16 quantities are fixed, it is straightforward to compute the EoS by using the energy density

$$
\begin{aligned}
\varepsilon= & \frac{1}{2} m_{\sigma}^{2} \sigma^{2}+\frac{1}{2} m_{\sigma^{*}}^{2} \sigma^{* 2}+\frac{1}{2} m_{\omega}^{2} \omega_{0}^{2}+\frac{1}{2} m_{\phi}^{2} \phi_{0}^{2}+\frac{1}{2} m_{\rho}^{2} \rho_{03}^{2}+U_{\sigma}^{\mathrm{QHD}} \\
& +\sum_{B} \frac{2 J_{B}+1}{2 \pi^{2}} \int_{0}^{k_{B}}\left[k^{2}+m_{B}^{* 2}\right]^{1 / 2} k^{2} d k+\sum_{l} \frac{1}{\pi^{2}} \int_{0}^{k_{l}}\left[k^{2}+m_{l}^{2}\right]^{1 / 2} k^{2} d k \\
& +m_{K}^{*} \rho_{K},
\end{aligned}
$$



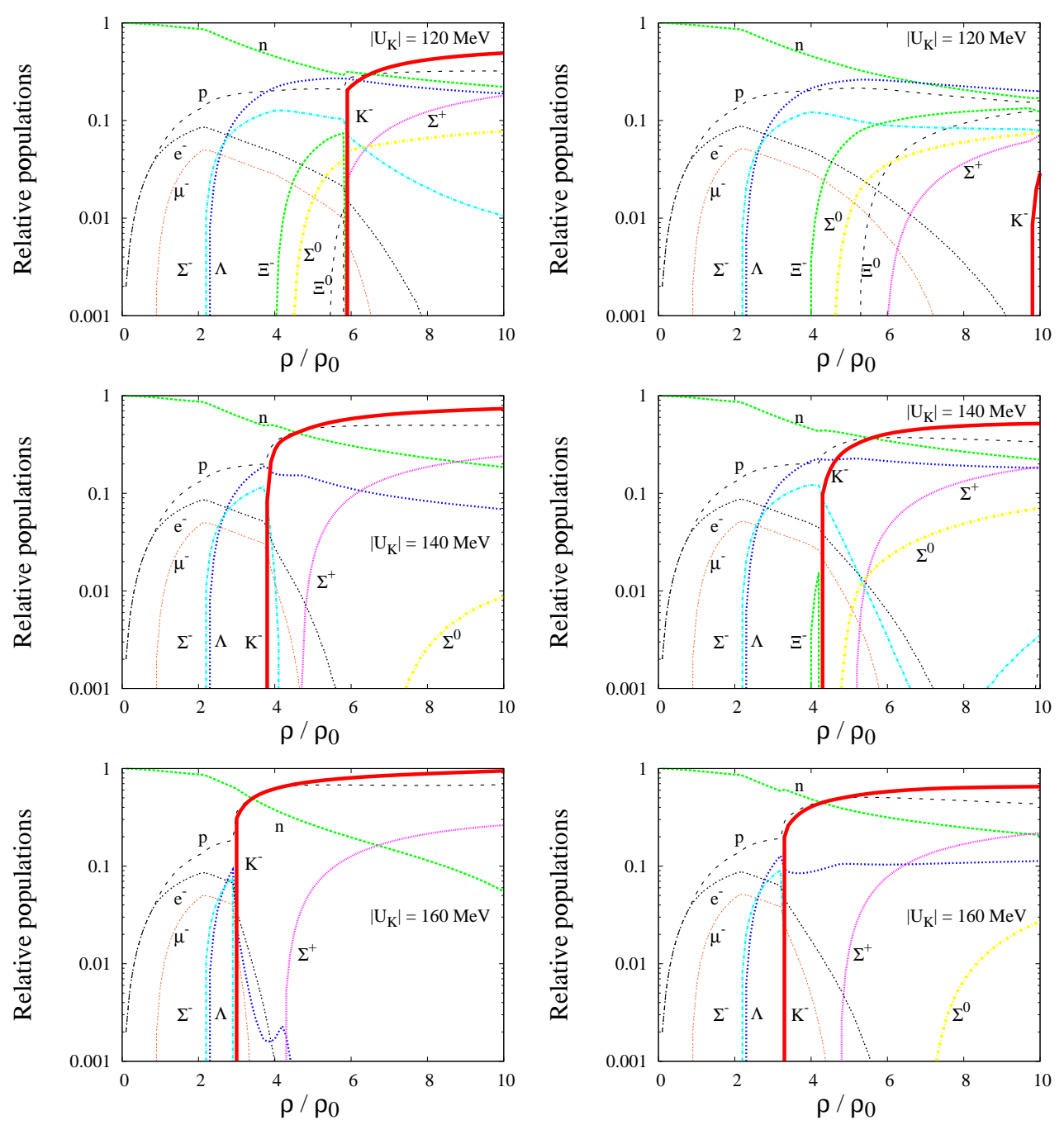

Figure 1: Compositions of neutron star matter from the MQMC (left panels) and the QHD (right panels) models.

and the pressure

$$
\begin{aligned}
P= & -\frac{1}{2} m_{\sigma}^{2} \sigma^{2}-\frac{1}{2} m_{\sigma^{*}}^{2} \sigma^{* 2}+\frac{1}{2} m_{\omega}^{2} \omega_{0}^{2}+\frac{1}{2} m_{\phi}^{2} \phi_{0}^{2}+\frac{1}{2} m_{\rho}^{2} \rho_{03}^{2}-U_{\sigma}^{\mathrm{QHD}} \\
& +\frac{1}{3} \sum_{B} \frac{2 J_{B}+1}{2 \pi^{2}} \int_{0}^{k_{B}} \frac{k^{4} d k}{\left[k^{2}+m_{B}^{* 2}\right]^{1 / 2}}+\frac{1}{3} \sum_{l} \frac{1}{\pi^{2}} \int_{0}^{k_{l}} \frac{k^{4} d k}{\left[k^{2}+m_{l}^{2}\right]^{1 / 2}} .
\end{aligned}
$$

Note that $U_{\sigma}^{\mathrm{QHD}}$ in $\varepsilon$ and $P$ is also only for the QHD and it is to be absent for the MQMC model.

\section{Results}

Fig. ${ }_{-1}^{1} \overline{1}$ shows the relative population (the ratio of the density of a particle to the total baryon density) in the neutron star matter up to $10 \rho_{0}$. The left panels show the results for the MQMC 

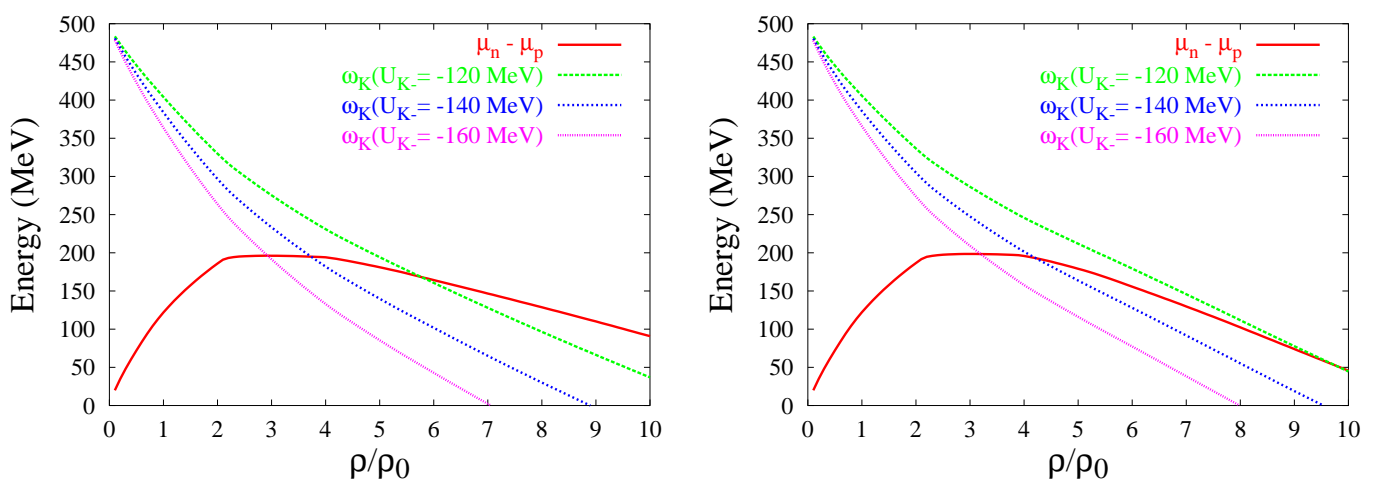

Figure 2: Kaon energy $\omega_{K}$ with $\left|U_{K^{-}}\right|=120 \mathrm{MeV}$ (dashed), $140 \mathrm{MeV}$ (dotted) and $160 \mathrm{MeV}$ (dot-dashed) is compared with $\mu_{n}-\mu_{p}$ (solid). At densities where $\omega_{K}$ and $\mu_{n}-\mu_{p}$ intersect, kaon starts to condense. The left panel is the result from the MQMC model and the right from the QHD.
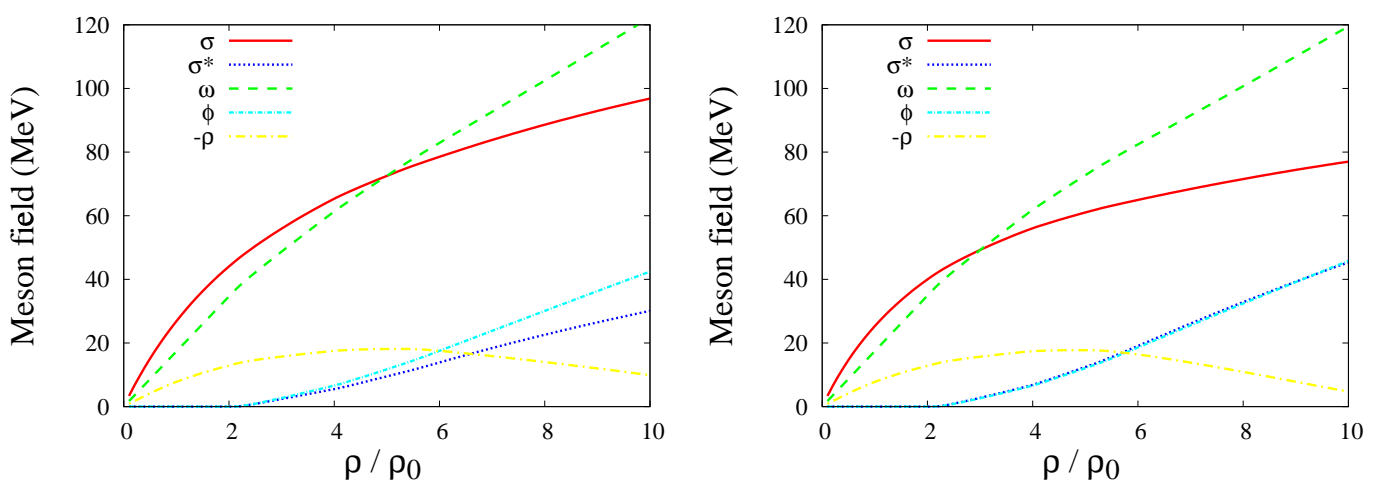

Figure 3: Meson fields in MeV from the MQMC (left) and the QHD (right).

model and the right for the QHD model. The two models consistently show that the onset density of the kaon condensation ( $\rho_{\text {crit }}$ ) becomes smaller with a larger $\left|U_{K^{-}}\right|$value. A larger $\left|U_{K^{-}}\right|$gives us a larger $g_{\sigma K}$, and thus leads to a faster decrease of $m_{K}^{*}$. A smaller $m_{K}^{*}$ makes $\omega_{K}$ value smaller, and consequently the chemical equilibrium condition for the kaon condensation, Eq. (2.2.24) is satisfied at a lower density. Fig. $\sum_{-1}^{2}$ illustrates this feature. The left (right) panel shows $\omega_{K}$ and $\mu_{n}-\mu_{p}$ from the MQMC (QHD) model. $\rho_{\text {crit }}$ is determined by the intersection of the curve for $\omega_{K}$ with the curve for $\mu_{n}-\mu_{p}$. When $U_{K^{-}}$changes, only $g_{\sigma K}$ changes among the variables that determine $\omega_{K}$. Therefore Fig. 2 shows the role of $U_{K^{-}}$in the determination of $\rho_{\text {crit }}$. The larger $\left|U_{K^{-}}\right|$is, the smaller $\rho_{\text {crit }}$ is.

Another common feature of the two models is that regardless of $\rho_{\text {crit }}$, once the kaon is created, its density piles up very quickly, and easily overwhelms the population of the hyperons and even

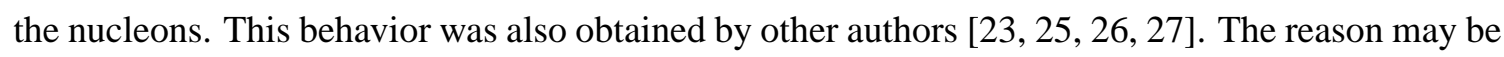
partly attributed to the opposite signs of the $\omega$-meson terms in the energy of $K^{-}$and octet baryons; negative for the former and positive for the latter. In Fig. $\overline{3}_{p}^{\prime}$ we plot the meson fields calculated from the MQMC (left) and the QHD (right) model as a function of $\rho$. >From the figure, one can easily 


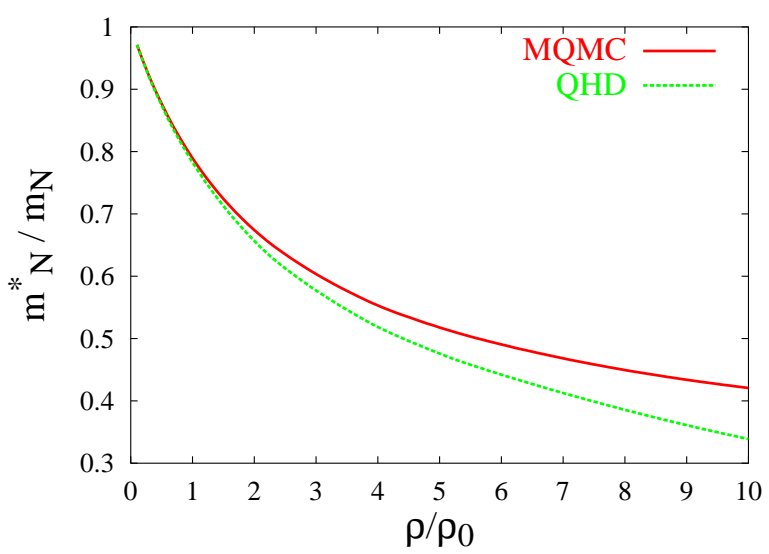

Figure 4: Effective mass of the nucleon.

deduce that the $\omega$-meson is a significant contributor to the energy of $K^{-}$and octet baryons. Since the $\omega$-meson contribution to $\omega_{K}$ is smaller than the hyperons by the order of $g_{\omega}^{q} \omega_{0}$, once the kaon is condensed, ground state strongly favors kaons than the hyperons, and this leads to fast increase of the kaon density. Since negatively charged hyperons compete directly with the kaon in the charge neutrality condition, they are highly suppressed by the presence of the kaon, and they disappear or are not created at all as soon as the kaon condensation sets in. Positively charged hyperons, on the other hand, undergo opposite influence from the kaon condensation: Creation density of $\Sigma^{+}$ gets lowered as $\left|U_{K^{-}}\right|$increases. Due to the charge neutrality, large abundance of the kaon leads to enhancement of the proton density, and it facilitates the chemical equilibrium condition of the positively charged hyperons.

Let us now discuss different aspects of the two models. Firstly the MQMC model predicts $\rho_{\text {crit }}$ to be smaller than that of the QHD for a given $\left|U_{K^{-}}\right|$value. For $\left|U_{K^{-}}\right|=120,140$ and $160 \mathrm{MeV}, \rho_{\text {crit }}$ values are $5.9 \rho_{0}, 3.8 \rho_{0}$ and $3.0 \rho_{0}$ in the MQMC model, respectively, while $\rho_{\text {crit }}$ is $9.8 \rho_{0}, 4.3 \rho_{0}$ and $3.3 \rho_{0}$ in the QHD model. Secondly, the MQMC model predicts larger population of the kaon than the QHD for a given $\left|U_{K^{-}}\right|$value. Fig. ${ }_{2}^{2}$ il illustrates the origin of the difference in $\rho_{\text {crit }}$ values from the two models. With a given $U_{K^{-}}$value, $\omega_{K}$ in the MQMC model decreases faster than that in the QHD because $\sigma$-meson field is larger in the MQMC than in the QHD, as seen in Fig. $\overline{3}_{1}^{\prime}$ Another reason for the difference in $\rho_{\text {crit }}$ can be found in the behavior of $\mu_{n}-\mu_{p}$. Roughly speaking, up to $\rho \sim 4 \rho_{0}, \mu_{n}-\mu_{p}$ behaves similarly in both models, but as density increases further, it drops down more rapidly in the QHD model. The reason, as shown in Fig. 幽, is mainly due to the behavior of $m_{N}^{*}$, which decreases more rapidly in the QHD model than in the MQMC. Larger population of the kaons in the MQMC model can be understood on the same ground. With smaller $\omega_{K}$ and larger effective mass of hyperons, the chemical equilibrium condition is more easily satisfied by the kaons than the hyperons. This suppresses the number of hyperons while increasing the density of the kaons.

The comparison of the population calculated from the MQMC and the QHD models indicates that the same inputs such as the saturation properties and $\left|U_{K^{-}}\right|$can result in quite different composition profile at high densities depending on the model. One reason for this difference may be 
said to be different properties of each model at high densities such as the role of self interactions of exchanged mesons, which are not controlled at saturation density in the present consideration. More direct information at high densities from either experiments or reliable calculation from more fundamental theory would help to reduce the present uncertainties.

\section{Conclusion}

Using the modified quark-meson coupling model, we have obtained the composition profile of neutron star matter, focusing on the role of the strange particles such as hyperons and kaons. Motivated by recent theoretical predictions of deeply bound kaonic states [i] $\left.{ }_{1}^{1} \overline{1}{ }_{1}^{\prime}\right]$ and the subsequent observations of interesting peaks found in KEK, BNL and DA $\Phi N E$ experiments, special attention is paid to the effect of large kaon optical potential $U_{K^{-}}$on the neutron star matter. By varying the value of $U_{K^{-}}$, we have investigated how the onset density of the kaon condensation and the composition of the stellar matter change. Employing a QHD model that satisfies the same saturation conditions as the MQMC model, we investigated the model dependence of the results.

We observed two common features in the models. First, a larger $\left|U_{K^{-}}\right|$produces a smaller onset density of the kaon condensation. This behavior is easily understood from the relation between $U_{K^{-}}$ and $g_{\sigma K}$ together with the role of $g_{\sigma K}$ to the energy of the kaon $\omega_{K}$. Secondly, the number of kaons increases very quickly, and suppresses the number of hyperons, especially negatively charged ones very strongly. This behavior is due to the different sign of the $\omega$-meson in energy of kaon and baryon; negative for the former and positive for the latter.

On the other hand, some model dependence was also observed. The onset density of the kaon condensation is smaller in MQMC model than in QHD model with the same $U_{K^{-}}$value, and the number of kaons also differs at a given density. These differences can be attributed to properties of the kaon and the baryon. Self-consistency condition gives different behavior of meson fields in each model. The effective mass (and consequently the chemical potential) of a baryon as a point particle differs substantially from that as a composite system as density becomes larger. This indicates that saturation conditions, which provide good starting point for the dense matter, don't have much control over the high density state. For instance, in this work we fixed $g_{\sigma K}$ with a given $U_{K^{-}}$value and the remaining kaon-meson coupling constants are determined by a simple quark counting rule.

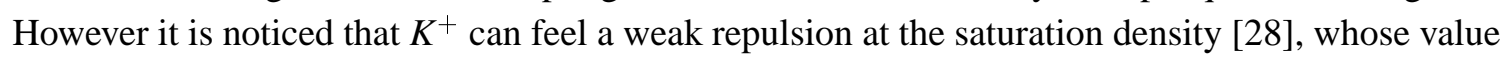
is about $+10 \mathrm{MeV}$. In our scheme, optical potential of $K^{+}$is given as $U_{K^{+}}=-g_{\sigma K} \sigma+g_{\omega K} \omega_{0}$. If we assume a value for $U_{K^{+}}$at the saturation density, together with a given $U_{K^{-}}, g_{\sigma K}$ and $g_{\omega K}$ can be fixed uniquely. With $g_{\sigma K}$ and $g_{\omega K}$ thus determined, $\omega_{K}$ will behave differently from what we have in this work since the contribution of $\omega$-meson will behave differently from the $\sigma$-meson at high densities. Therefore it can be said that more direct information on the high density matter will be important to understand the state of matter that constitutes the neutron star.

In this work we have considered the composition of the neutron star matter only. Equation of state, inserted in the Tolman-Oppenheimer-Volkoff equation, will give us a direct relationship between the mass and the radius of the neutron star. Calculation of the equation of state and solving the Tolman-Oppenheimer-Volkoff equation are in progress. 


\section{Acknowledgments}

This work was supported by the Basic Research Program of the Korea Science \& Engineering Foundation (R01-2005-000-10050-0) and by APCTP 2005 Topical Program. The authors thank Prof. Y. Akaishi for useful discussions.

\section{References}

[1] S. I. A. Garpman, N. K. Glendenning and Y. J. Karant, Nucl. Phys. A322, 382 (1979).

[2] A. B. Migdal, Rev. Mod. Phys. 50, 107 (1978).

[3] D. B. Kaplan and A. E. Nelson, Phys. Lett. B175, 57 (1986).

[4] A. R. Bodmer, Phys. Rev. D 4, 1601 (1971).

[5] N. Itoh, Prog. Theor. Phys. 44, 291 (1970).

[6] G. Baym and S. A. Chin, Phys. Lett. B62, 241 (1976).

[7] B. D. Keister and K. S. Kisslinger, Phys. Lett. B64, 117 (1976).

[8] J. Schaffner-Bielich, V. Koch and M. Effenberger, Nucl. Phys. A669 153 (2000).

[9] A. Ramos and E. Oset, Nucl. Phys. A671, 481 (2000).

[10] A. Cieply, E. Friedman, A. Gal and J. Mares, Nucl. Phys. A696 173 (2001).

[11] E. Friedman, A. Gal and C. J. Batty, Nucl. Phys. A579, 578 (1994).

[12] N. Kaiser, P.B. Siegel and W. Weise, Nucl. Phys. A594 325 (1995).

[13] C. J. Batty, E. Friedman, A. Gal, Phys. Rep. 287, 385 (1997).

[14] T. Yamazaki and Y. Akaishi, Phys. Lett. B535 70 (2002); Y. Akaishi and T. Yamazaki, Phys. Rev. C 65, 044005 (2002).

[15] T. Suzuki et al., Phys. Lett. B597, 263 (2004).

[16] T. Suzuki et al., Nucl. Phys. A754, 375c (2005).

[17] T. Kishimoto et al., Nucl. Phys. A754, 383c (2005).

[18] M. Agnello et al., Phys. Rev. Lett. 94, 212303 (2005).

[19] X. Jin and B. K. Jennings, Phys. Lett. B374, 13 (1996); Phys. Rev. C 54, 1427 (1996).

[20] S. Pal, M. Hanauske, I. Zakout, H. Stöcker and W. Greiner, Phys. Rev. C 60, 015802 (1999).

[21] S. Fleck, W. Bentz, K. Shimizu and K. Yazaki, Nucl. Phys. A510, 731 (1990).

[22] K. Saito and A. W. Thomas, Phys. Lett. B327, 9 (1994).

[23] N. K. Glendenning and J. Schaffner, Phys. Rev. C 60, 025803 (1999).

[24] T. A. Armstrong et al., WA76 Collaboration, Z. Phys. A 51, 351 (1991).

[25] R. Knorren, M. Prakash and P. J. Ellis, Phys. Rev. C 52, 3470 (1995).

[26] S. Banik and D. Bandyopadhyay, Phys. Rev. C 64, 055805 (2001).

[27] D. P. Menezes, P. K. Panda and C. Providencia, Phys. Rev. C 72, 035802 (2005).

[28] G. Q. Li, C.-H. Lee and G. E. Brown, Phys. Rev. Lett. 79, 5214 (1997). 